\title{
Heterogeneidade de Variâncias nos Grupos Genéticos Formadores da Raça Canchim ${ }^{1}$

\author{
Carlos Antonio Lopes de Oliveira ${ }^{2}$, Elias Nunes Martins ${ }^{3}$, Alfredo Ribeiro de Freitas ${ }^{4}$,
} Maurício Mello de Alencar ${ }^{4}$
}

\begin{abstract}
RESUMO - Foram estimados componentes de variância para os pesos aos 365 e 550 dias de idade, de animais dos grupos genéticos, 1/2 Charolês-Zebu, 3/4 Zebu-Charolês e 5/8 Charolês-Zebu, utilizados no processo de formação da raça Canchim, com o objetivo de verificar a ocorrência de variâncias heterogêneas entre os grupos genéticos e seu possível efeito na classificação dos animais, na avaliação genética. As estimativas foram obtidas utilizando-se o sistema MTDFREML. A estratégia utilizada para estimar os componentes de variâncias para as duas características foi considerar a expressão das características em cada grupo genético como uma característica diferente. Portanto, foram testados diferentes modelos com intenção de verificar diferenças nas estimativas dos componentes de variâncias dos diferentes grupos genéticos. As estimativas dos componentes de variância e as classificações dos melhores animais foram diferentes, para os modelos unicaracter e denominado tricaracter. As porcentagens de indivíduos comuns, no processo de avaliação genética considerando ou não a ocorrência de variâncias heterogêneas, tomando-se todo o conjunto de dados e separadamente machos e fêmeas, foram, respectivamente, 83,$7 ; 86,6$ e 89,3\% para peso aos 365 dias e 69,$3 ; 75,5$ e 72,5\% para peso aos 550 dias. Os resultados indicaram a existência de variâncias heterogêneas nos grupos genéticos participantes da formação da raça Canchim para peso aos 365 e 550 dias.
\end{abstract}

Palavras-chave: avaliação genética, heterogeneidade de variâncias, raça Canchim

\section{Heterogeneity of Variances in the Genetic Groups Formed by the Canchim Breed}

ABSTRACT - Variance components were estimated for the characteristic weights at 365 and 550-days of age of animals from genetic groups used on Canchim breed development. The objective was to verify the occurrence of heterogeneity variance among the genetic groups and the its possible effect during the ranking of animals, in the genetic evaluation. The estimates were obtained for the software MTDFREML. The strategy used to estimate the components of variance for both characteristics was to consider the expression of the characteristics in each genetic group as a different characteristic. The different models were tested in order to verify difference in the estimates of variance components for differents genetic groups. The estimates of variance components and classifications of the betters animals were different, for models one-trait and denominated three-trait. During the genetic evaluation process, the results were 83.7, 86.6 and $89.3 \%$ of the selected individuals there were two common classifications for the weight at 365 days, taking into account the entire set of data, males and females, respectively. For the weight at 550 days, $75.5,72.5$ and $69.3 \%$ were common between the two classifications for males, females and for the whole set of data, respectively. The results showed an existence of heterogeneous variances for the genetic groups used during the formation of the Canchim breed, for weight 365 and 550 days of age.

Key Words: Canchim breed, genetic evaluations, heterogeneity of variances

\section{Introdução}

A heterogeneidade de variâncias dentro de níveis de efeitos fixos tem sido verificada por diversos autores, em se tratando de características de produção de leite e desenvolvimento ponderal (WINKELMAN e SCHAEFFER, 1988; DONG e MAO, 1990; TORRES, 1998; RODRIGUEZALMEIDA et al., 1995; CREWS e FRANKE, 1998). De VEER e Van VLECK (1987) e IBAÑEZ et al. (1996) sugerem, como fatores responsáveis pela heterocedasticidade, região, ano de nascimento, nível de manejo, variabilidade genética dentro do rebanho e nível de produção.

Em gado de corte, variâncias heterogêneas têm sido verificadas para grupos genéticos diferentes, conforme CREWS e FRANKE (1998), que observaram heterocedasticidade para diferentes "graus de sangue" Brahman, para características de carcaça e RODRIGUEZ-ALMEIDA et al. (1995), que encontraram variâncias heterogêneas de reprodutor e fenotípicas, para pesos aos 220 e 365 dias, em animais

\footnotetext{
${ }^{1}$ Parte da dissertação de Mestrado do primeiro autor, apresentada à Universidade Estadual de Maringá - UEM - PR

2 Professor da Universidade Estadual do Mato Grosso do Sul - Aquidauana - MS. E.mail: calolive@hotmail.com

3 Professor da Universidade Estadual de Maringá - PR. E.mail: enmartins@uem.br

4 Pesquisadores da EMBRAPA-CPPSE, São Carlos - SP.
} 
filhos de touros de 22 raças diferentes acasaladas com fêmeas Aberdeen angus e Hereford.

Segundo VINSON (1987), a heterogeneidade de variâncias deve ser considerada no processo de avaliação genética. Com base nisto, NUNEZDOMINGUEZ et al. (1995) e CREWS e FRANKE (1998) encontraram alterações de postos quando compararam classificações fornecidas por modelos que consideravam ou não a existência de variâncias heterogêneas, em características de crescimento e de carcaça de animais de diferentes grupos genéticos.

Os processos de formação de raças sintéticas utilizam tanto a seleção quanto o cruzamento como ferramentas. Assim, é provável que as diferentes gerações caracterizadas pelos diversos grupos genéticos apresentem heterogeneidade de variância.

Os objetivos do presente trabalho foram testar diferentes modelos para estimação dos componentes de variância para as características pesos aos 365 e 550 dias de idade, considerando-se os diferentes grupos genéticos formadores da raça Canchim, e avaliar a ocorrência da estimativas discordantes destes componentes de variância e seu efeito na classificação dos animais.

\section{Material e Métodos}

Foi utilizado um conjunto de dados contendo informações dos animais formadores da raça Canchim, cedido pela EMBRAPA-CPPSE (Empresa Brasileira de Pesquisa Agropecuária - Centro de Pesquisa de Pecuária do Sudeste), São Carlos - SP. Foram estimados os componentes de (co)variância para as características pesos aos 12 meses ajustado para 365 dias (P365) e peso aos 18 meses ajustado para 550 dias (P550), para animais 1/2 Charolês-Zebu, 3/4 Zebu-Charolês e 5/8 Charolês-Zebu, conforme o esquema de cruzamento de animais das raças Charolesa, Indubrasil, Guzerá e Nelore, para formação da raça Canchim (VIANA, et al., 1962).

Os dados continham informações de 407 animais, 1/2 Charolês-Zebu (GG1), 567 mestiços, 3/4 ZebuCharolês (GG2) e 814 animais 5/8 Charolês-Zebu (GG3) para P365 e na mesma ordem, 291, 386 e 741 animais para P550 nos diferentes grupos genéticos.

Foram estimados os componentes de variância para as características P365 e P550 em análises unicaracter. Para estimar os componentes de variância dos diferentes grupos genéticos, utilizou-se como estratégia considerar como características diferentes os pesos aos 365 dias e 550 dias nos três grupos genéticos. Assim, foram realizadas análises denominadas tricaracter (tricaracter) para P365 e P550, quando a expressão de uma das características, foi considerada nos três grupos genéticos separadamente.

O modelo considerado nas análises unicaracter, para as duas características é dado a seguir, e contém apenas efeito de grupo genético que o diferencia do modelo para as análises tricaracter,

$$
\begin{gathered}
\mathrm{y}_{i j k l m n}=\mu+\mathrm{g}_{i}+\mathrm{s}_{j}+\mathrm{an}_{k}+\mathrm{ea}_{l}+\mathrm{b}_{1}\left(i \bar{v}_{m^{-}}\right)+\mathrm{b}_{2}\left(i \bar{v}_{m^{-}}\right) \\
2+\mathrm{a}_{i j k l m n}+\mathrm{e}_{i j k l m n}
\end{gathered}
$$

em que: $y_{i j k l m n}$ são as observações referentes aos pesos ajustados para 365 e 550 dias; $\mu$ é uma constante associada a toda observação; $\mathrm{g}_{i}$ é o efeito do grupo genético i; i - 1, 2, 3; s é o efeito do sexo j; j = 1, 2; $\mathrm{an}_{k}$ é o efeito do ano de nascimento $\mathrm{k}, \mathrm{k}=1949, \ldots$, 1969; ea é $_{l}$ o efeito da estação do ano 1 , em que o animal nasceu, $1=1,2,3$ e 4; $b_{1}$ e $b_{2}$ são coeficientes de regressão dos termos linear e quadrático dos pesos aos 365 dias ou 550 dias, em função da idade da vaca ao parto; $i v_{m}$ é a idade da vaca $\mathrm{m}$, ao parto; $i v$ é a média de idade das vacas ao parto; $\mathrm{a}_{i j k l m n}$ é o efeito aleatório associado ao efeito genético aditivo de cada animal; $\mathrm{e}_{j k l m n}$ é o erro aleatório associado a cada observação.

Os modelos para estimação dos componentes de variância diferiram de acordo com o tipo de análise, uni ou tricaracter. Nas análises tricaracter, não se considerou o efeito fixo de grupo genético. O modelo utilizado nas diferentes análises pode ser representado matricialmente da seguinte forma:

$$
\left[\begin{array}{l}
y_{1} \\
y_{2} \\
y_{3}
\end{array}\right]=\left[\begin{array}{ccc}
X_{1} & 0 & 0 \\
0 & X_{2} & 0 \\
0 & 0 & X_{3}
\end{array}\right]\left[\begin{array}{l}
\beta_{1} \\
\beta_{2} \\
\beta_{3}
\end{array}\right]+\left[\begin{array}{ccc}
Z_{1} & 0 & 0 \\
0 & Z_{2} & 0 \\
0 & 0 & Z_{3}
\end{array}\right]\left[\begin{array}{l}
a_{1} \\
a_{2} \\
a_{3}
\end{array}\right]+\left[\begin{array}{l}
e_{1} \\
e_{2} \\
e_{3}
\end{array}\right][2]
$$

em que: $y_{1}, y_{2}$ e $y_{3}$ são os vetores de observações, para P365 ou P550, referentes aos animais dos grupos genéticos GG1, GG2 e GG3, respectivamente; $X_{1}, X_{2}$ e $X_{3}$ são as matrizes de incidência dos efeitos fixos, para P365 ou P550, referentes aos animais dos grupos genéticos GG1, GG2 e GG3, respectivamente; $\beta_{1}, \beta_{2}$ e $\beta_{3}$ são os vetores dos efeitos fixos, para P365 ou P550, referentes aos animais dos grupos genéticos GG1, GG2 e GG3, respectivamente; $Z_{1}, Z_{2}$ e $Z_{3}$ são as matrizes de incidência dos efeitos aleatórios para P365 ou P550, referentes aos animais dos grupos genéticos GG1, GG2 e GG3, respectivamente; $\mathrm{a}_{1}, \mathrm{a}_{2}$ e $a_{3}$ são os vetores dos efeitos genéticos diretos, para P365 ou P550, referentes aos animais dos grupos genéticos GG1, GG2 e GG3, respectivamente; $\mathrm{e}_{1}, \mathrm{e}_{2}$ e $\mathrm{e}_{3}$ são os vetores dos erros aleatórios, para $\mathrm{P} 365$ ou P550, referentes aos animais dos grupos 
1214 Rev. bras. zootec.

genéticos GG1, GG2 e GG3, respectivamente. No caso das análises unicaracter, o vetor das observações de P365 e P550, dos vetores dos efeitos fixos, genéticos diretos e dos erros aleatórios não foram diferenciados para os três grupos genéticos, sendo da mesma forma para as matrizes de incidência dos efeitos fixos e aleatórios.

A caracterização das distribuições dos fatores, de efeito aleatório, contidos no modelo estatístico e as matrizes que representam componentes de variância estão representadas da mesma forma como descrito por TORRES (1998).

Para estimação dos componentes de (co)variância, foi utilizado o sistema computacional MTDFREML Multiple Trait Derivative Free Restricted Maximum Likelihood desenvolvido por BOLDMAN et al. (1993). Este sistema utiliza conceitos do método da máxima verossimilhança restrita, considerando, para estimação dos componentes de (co)variância, apenas a parte referente aos efeitos aleatórios da função densidade de probabilidade das observações, referente ao modelo unicaracter que é dada por,

$$
f(y)=\frac{1}{(2 \pi)^{\rho / 2}\left|K\left(Z G Z^{\prime}+R\right) K^{\prime}\right|^{1 / 2}} e^{-\frac{1}{2}\left(y^{\prime} P y\right)}
$$

em que: $p=$ é o posto da matriz projetor ortogonal da parte aleatória das observações; $K$ = é uma matriz que estabelece contrastes linearmente independentes entre as partes aleatórias das observações; $\mathrm{P}=$ é a matriz projetor ortogonal da parte aleatória das observações, dada por:

$$
P=V^{-1}-V^{-1} X^{\prime}\left(X^{\prime} V^{-1} X\right)^{-} X^{\prime} V^{-1}
$$

Após a transformação logarítmica e devidas manipulações matemáticas, obtém-se a função a ser avaliada:

$-2 \log L=$ constante $+\log |G|+\log |R|+\log |C|+y^{\prime} P y$

em que: $\mathrm{C}$ é a matriz dos coeficientes das equações do modelo misto considerado.

No método DFREML, os componentes de (co)variância são estimados utilizando-se o método livre de derivadas proposto por Graser et al. (1986) conforme BOLDMAN et al. (1993), que consiste num processo de procura dos valores que minimizam a função apresentada em [4], realizando avaliações dos valores assumidos pela função de verossimilhança em cada iteração, por meio do método Simplex. Para todas as análises realizadas neste trabalho, o critério de convergência utilizado foi à variância do simplex menor ou igual a $10^{-12}$.
Após a estimação dos componentes de (co)variância, foi aplicado o teste da razão de verossimilhança (MOOD et al., 1974), nos modelos uni e tricaracter, para identificar o modelo mais apropriado, sendo a diferença obtida entre os logaritmos dos valores da função de verossimilhança $(-2 \log L)$, à convergência, dos modelos com maior e menor número de parâmetros estimados, testada contra os valores obtidos na distribuição de $\chi^{2}$ (qui-quadrado) com $\mathrm{P}<0,005$ e graus de liberdade dados pela diferença do número de parâmetros estimados pelos modelos em questão. Os valores de $-2 \log \mathrm{L}$ foram obtidos dos resultados do programa MTDRUN, parte do sistema MTDFREML.

Após a verificação do modelo mais adequado, se o uni ou tricaracter, foram agrupados dois a dois os grupos genéticos, o que permitiu identificar a existência de modelos mais adequados resultantes da agregação de grupos genéticos diferentes.

Diante disso, foram realizados testes da razão da verossimilhança entre os modelos denominados bicaracter (bicaracter), resultado da agregação dos grupos genéticos dois a dois, em possíveis grupos de heterogeneidade de variância e os modelos tricaracter.

Em função dos resultados do teste da razão de verossimilhança, para as duas características, os animais foram classificados de acordo com os valores genéticos, obtidos através da metodologia BLUP (Best Linear Unbiased Prediction), proposto por HENDERSON (1975), utilizando o modelo [2] considerando estimativas de componentes de variância diferentes para cada grupo genético. Além disso, os animais foram classificados, utilizando-se as estimativas obtidas pelo modelo unicaracter. Foram obtidas três classificações por característica e por modelo, sendo classificados machos e fêmeas separadamente e todo o conjunto de valores genéticos de maneira geral. Sobre estas classificações, foi aplicado o teste de correlação de Spearman conforme SPIEGEL (1977) e SAMPAIO (1998), para verificar se houve alteração de postos, entre as classificações fornecidas pelos modelos uni, bi ou tricaracter, de acordo com os resultados do teste da razão de verossimilhança.

Além disso, foram atribuídas pressões de seleção de 10 e $30 \%$ para machos e fêmeas e de $10 \%$ para o conjunto total de valores genéticos. Em seguida, verificou-se a porcentagem de indivíduos selecionados comuns, entre o modelo unicaracter e o modelo mais apropriado, indicado pelo teste da razão de verossimilhança, para as duas características. 


\section{Resultados e Discussão}

Verificou-se que o modelo tricaracter apresentou menores valores de -2log L, para as duas características. O teste da razão de verossimilhança indicou que o modelo considerando os três grupos genéticos separadamente foi o mais adequado, para as duas características $(\mathrm{p}<0,05)$, quando comparado com os modelos uni e bicaracter.

As estimativas dos componentes de variância e herdabilidades obtidos pelos modelos uni e tricaracter estão sumarizadas nas Tabelas 1 e 2. Verificaram-se valores discordantes das estimativas obtidas nos modelos uni e tricaracter e entre os diferentes grupos genéticos, para as duas características.

Os resultados indicaram o grupo genético $5 / 8$ Charolês-Zebu como o de maior variabilidade genética, residual e fenotípica e o grupo genético $3 / 4$ Zebu-

Charolês como o de menor variância residual, para as duas características.

As maiores estimativas de herdabilidade foram obtidas para o grupo genético $3 / 4$ Zebu-Charolês, sendo superiores às fornecidas pelo modelo unicaracter, para as duas características. Com exceção da estimativa de herdabilidade para o grupo genético $1 / 2$ Charolês-Zebu na característica $\mathrm{P} 365$, todas as estimativas resultantes do modelo tricaracter foram diferentes das fornecidas pelo modelo unicaracter.

Considerando as correlações genéticas, conforme Tabela 3, verificou-se que os grupos genéticos que apresentaram maior correlação foram $0 \quad 1 / 2$ Charolês-Zebu com o 3/4 Zebu-Charolês e 1/2 Charolês-Zebu com 5/8 Charolês-Zebu, para as características peso aos 365 e 550 dias, respectivamente, indicando que não há, neste caso, um padrão da associação genética entre os grupos genéticos

Tabela 1 - Estimativas de componentes de variância e herdabilidade, obtidas com modelos unicaracter e denominado tricaracter, para a característica P365

Table 1 - Estimates of variance components and heritability, on a one-trait e three-traits models for weight at 365 days

\begin{tabular}{lccr}
\hline $\begin{array}{l}\text { Estimativas } \\
\text { Estimates }\end{array}$ & $\begin{array}{c}\text { Unicaracter } \\
\text { One-trait }\end{array}$ & \multicolumn{2}{c}{$\begin{array}{c}\text { Tricaracter } \\
\text { Three-traits }\end{array}$} \\
\hline $\begin{array}{l}\text { Variância genética aditiva } \\
\begin{array}{l}\text { Aditive genetic variance } \\
\text { Variância residual }\end{array}\end{array}$ & 452,15 & $\mathrm{GG}^{1}$ & $\mathrm{GG}^{2}$ \\
$\begin{array}{l}\text { Residual variance } \\
\text { Variância fenotípica }\end{array}$ & 692,44 & 416,825 & 377,581 \\
$\begin{array}{l}\text { Phenotypic variance } \\
\begin{array}{l}\text { Herdabilidade } \\
\text { Heritability }\end{array}\end{array}$ & 1144,58 & 627,356 & 389,144 \\
\hline
\end{tabular}

1 GG1: $1 / 2$ Charolês-Zebu 2. GG2: 3/4 Zebu-Charolês 3. GG3: 5/8 Charolês-Zebu.

GG1:1/2 Charolais-Zebu 2. GG2: 3/4 Zebu-Charolais 3. GG3: 5/8 Charolais-Zebu.

Tabela 2 - Estimativas de componentes de variância e herdabilidade, obtidas com modelos unicaracter e denominado tricaracter, para característica P550

Table 2 - Estimates of variance components and heritability, on a one-trait e three-traits models for weight at 550 days

\begin{tabular}{|c|c|c|c|c|}
\hline \multirow[t]{2}{*}{$\begin{array}{l}\text { Estimativas } \\
\text { Estimates }\end{array}$} & \multirow[t]{2}{*}{$\begin{array}{c}\text { Unicaracter } \\
\text { One-trait }\end{array}$} & \multicolumn{3}{|c|}{$\begin{array}{l}\text { Tricaracter } \\
\text { Three-traits }\end{array}$} \\
\hline & & GG1 $^{1}$ & $\mathrm{GG} 2^{2}$ & GG3 $^{3}$ \\
\hline $\begin{array}{l}\text { Variância genética aditiva } \\
\text { Aditive genetic variance }\end{array}$ & 509,83 & 124,471 & 457,336 & 671,05 \\
\hline $\begin{array}{l}\text { Variância residual } \\
\text { Residual variance }\end{array}$ & 692,44 & 747,486 & 536,965 & 1149,14 \\
\hline $\begin{array}{l}\text { Variância fenotípica } \\
\text { Phenotypic variance }\end{array}$ & 1144,58 & 871,957 & 994,301 & 1820,19 \\
\hline $\begin{array}{l}\text { Herdabilidade } \\
\text { Heritability }\end{array}$ & 0,34 & 0,14 & 0,46 & 0,37 \\
\hline
\end{tabular}

${ }^{1}$ GG1: $1 / 2$ Charolês-Zebu 2. GG2: 3/4 Zebu-Charolês 3. GG3: 5/8 Charolês-Zebu.

GG1:1/2 Charolais-Zebu 2. GG2: 3/4 Zebu-Charolais 3. GG3: 5/8 Charolais-Zebu. 
1216 Rev. bras. zootec.

Tabela 3 - Estimativas de correlações genéticas entre os grupos genéticos para peso aos 365 e 550 dias, em modelos tricaracter

Table 3 - Estimates of genetic correlations among genetic groups for weight at 365 and 550 days, on three-traits models

\begin{tabular}{lcc}
\hline $\begin{array}{l}\text { Características } \\
\text { Traits }\end{array}$ & $\begin{array}{c}\text { Grupos } \\
\text { genéticos } \\
\text { Genetic } \\
\text { groups }\end{array}$ & $\begin{array}{c}\text { Correlações } \\
\text { genéticas } \\
\text { Genetics } \\
\text { correlations }\end{array}$ \\
\hline $\begin{array}{l}\text { Peso aos 365 dias } \\
\text { Weight at 365 days }\end{array}$ & GG1-GG2 & 0,99 \\
& GG1-GG3 & 0,83 \\
Peso aos 550 dias & GG2-GG3 & 0,91 \\
Weight at 550 days & GG1-GG2 & 0,89 \\
& G1-GG3 & 1,00 \\
& GG2-GG3 & 0,84 \\
\hline
\end{tabular}

para as duas características. Todavia verificou-se ampla associação genética entre os grupos genéticos para as duas características.

As estimativas dos componentes de variância e dos parâmetros genéticos obtidos nas diversas análises realizadas neste trabalho, para peso aos 365 dias, são superiores às encontradas para animais Nelore, conforme ELER et al. (1989); ELER et al. (1995) e MAGNABOSCO et al. (1995), utilizando DFREML e Inferência Bayesiana. Deve-se ressaltar, no entanto, que nestes trabalhos os autores consideravam nos modelos a presença de efeito genético materno e de ambiente permanente.

Em relação a outros grupos genéticos, observou-se para peso aos 365 dias, que as estimativas de herdabilidade obtidas neste trabalho são inferiores às encontradas por FREITAS e VENCOVSKY (1993) e MASCIOLI et al. (1996) com animais Canchim e são semelhantes às fornecidas por GREGORY et al. (1995) para machos inteiros de 12 grupos genéticos diferentes.

Em se tratando de peso aos 550 dias, SILVA et al. (1987), LÔBO et al. (1995) e MAGNABOSCO et al. (1995), em animais Nelore, encontraram estimativas de herdabilidade inferiores às obtidas neste trabalho, quando foram consideradas as estimativas resultantes do modelo unicaracter e para os grupos genéticos 3/4 Zebu-Charolês e 5/8 Charolês-Zebu. Entretanto, MAGNABOSCO (1997) analisando dados de rebanhos de animais da raça Nelore, encontrou valores semelhantes aos obtidos neste trabalho.

Considerando outros grupos genéticos, MASCIOLI et al. (1996) encontraram, em animais Canchim, estimativas de herdabilidade superiores às encontradas neste trabalho. No entanto, MEYER et al. (1991), em animais mestiços Zebu, encontraram valores inferiores aos obtidos neste trabalho, utilizando DFREML em modelos bicaracter, considerando características de crescimento e reprodutivas.

Os valores de correlação de Spearman e os resultados dos testes aplicados (Tabela 4) demonstram correlação significativa $(\mathrm{P}<0,05)$ entre os postos das diferentes classificações. Além disso, verificou-se maior valor de correlação para a característica peso aos 365 dias, considerando as classificações de machos e fêmeas, separadamente e de todo o conjunto de dados (geral), quando comparado com os valores obtidos para o peso aos 550 dias. Este resultado indica que a desconsideração de diferentes componentes de variância para cada grupo genético resultou em menor associação entre as classificações dos animais, nos modelos uni e tricaracter, na característica peso aos 550 dias.

Ao serem atribuídas pressões de seleção de $10 \%$ para todo o conjunto de dados, $10 \%$ para machos e $30 \%$ para fêmeas, separadamente, verificou-se que as percentagens de indivíduos comuns às classificações para os dois modelos foram de 83,7, 86,6 e $89,3 \%$ dos indivíduos selecionados para peso aos 365 dias, considerando todo o conjunto de dados, machos e fêmeas, respectivamente.

Em relação à característica peso aos 550 dias, foram encontrados 75,5, 72,5 e 69,3\% de indivíduos comuns às classificações dos modelos unicaracter e denominado tricaracter, para machos, fêmeas e para todo o conjunto de dados, respectivamente. Tal fato pode ser explicado pela a maior discordância entre os valores estimados da variância genética aditiva para cada grupo genético na característica P550, quando comparado aos valores estimados para os grupos genéticos na característica P365 (Tabelas 1 e 2). Estes resultados corroboram o valor da correlação de Spearman, que indicou menor associação entre as classificações fornecidas pelos diferentes modelos, na característica peso aos 550 dias.

A aplicação de diferentes pressões de seleção indicou que os animais selecionados discordavam para os diferentes modelos, quando foram utilizados os modelos uni e tricaracter, ao considerar-se os valores genéticos absolutos (Tabela 5 e 6). No entanto, valores estimados para correlação de postos nos diferentes modelos e os percentuais de indivíduos comuns selecionados nos diferentes modelos com diferentes pressões de seleção, não permitem conclusões a respeito da influência da ocorrência de 
OLIVEIRA et al.

Tabela 4 - Valores de Correlação de Spearman, de t calculado e graus de liberdade, nas análises considerando ou não a heterogeneidade de variância, para todo o conjunto de dados (geral), machos e fêmeas separadamente, em P365 e P550

Table 4 - Values of Spearman correlations, $t$ value and degrees of freedom, in analyses considering or not the variances heterogeneous, for all observations (general), males and females, for weight at 365 and 550 days old

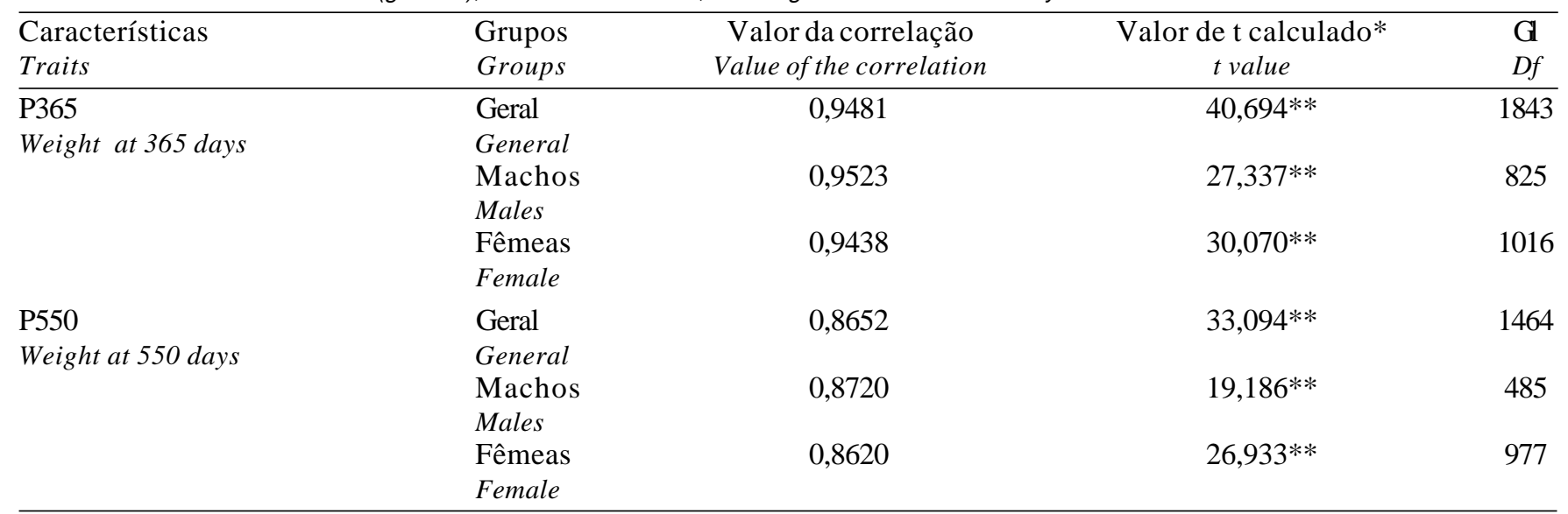

* Foram comparadas com o valor da distribuição de $t(0,005 ; ¥)$; ** Significativo $(\mathrm{P}<0,005)$.

${ }^{\star}$ Comparison with $t$-value (.005; ¥); ** Significant $(P<.005)$.

variâncias específicas sobre as classificações dos animais de diferentes grupos genéticos, principalmente para a característica peso aos 365 dias.

Procedimentos e resultados semelhantes ao obtidos neste trabalho podem ser encontrados no trabalho de CREWS e FRANKE (1998), com características de carcaça, em animais com diferentes "graus de sangue" Brahman, que verificaram valores de correlações entre 0,99 e 0,82 , para as classificações considerando ou não a existência de variâncias específicas para os diferentes graus de sangue. Estes autores salientam que correlações de postos menores que 0,90 , podem resultar em alterações nas classificações dos animais, quando se considera ou não a existência de variâncias específicas para cada grupo genético.

Em experimento com 12 raças de reprodutores, cruzados com fêmeas Aberdeen Angus e Hereford, NUÑEZ-DOMINGUES et al. (1995) encontraram que o modelo que considerava variâncias específicas para os grupos genéticos, apresentou melhor ajustamento. Verificaram, também, valores de correlações de postos que indicaram alteração nas classificações fornecidas pelos diferentes modelos testados.
Tabela 5 - Classificação dos animais para a característica P365 (Geral), considerando os modelos uni e tricaracter

Table 5 - Animals classifications for trait P365 (General), considering the one and three-trait models

\begin{tabular}{|c|c|c|c|c|}
\hline \multirow[b]{2}{*}{$\begin{array}{l}\text { Classificação } \\
\text { Ranking }\end{array}$} & \multicolumn{2}{|c|}{$\begin{array}{c}\text { Unicaracter } \\
\text { One-trait }\end{array}$} & \multicolumn{2}{|c|}{$\begin{array}{l}\text { Tricaracter } \\
\text { Three-trait }\end{array}$} \\
\hline & $\begin{array}{l}\text { Animal } \\
\text { Animal }\end{array}$ & $\begin{array}{l}\text { Sexo } \\
\text { Sex }\end{array}$ & $\begin{array}{l}\text { Animal } \\
\text { Animal }\end{array}$ & $\begin{array}{l}\text { Sexo } \\
\text { Sex }\end{array}$ \\
\hline 1 & 800072 & 1 & 800072 & 1 \\
\hline 2 & 1000169 & 1 & 1000169 & 1 \\
\hline 3 & 1000611 & 1 & 900165 & 2 \\
\hline 4 & 900165 & 2 & 900177 & 1 \\
\hline 5 & 900177 & 1 & 900157 & 2 \\
\hline 6 & 900191 & 2 & 900393 & 2 \\
\hline 7 & 1000600 & 1 & 1000186 & 2 \\
\hline 8 & 1000185 & 1 & 1000315 & 2 \\
\hline 9 & 900157 & 2 & 900066 & 2 \\
\hline 10 & 1000438 & 1 & 900170 & 1 \\
\hline 11 & 1000186 & 2 & 1000185 & 1 \\
\hline 12 & 1000315 & 2 & 900140 & 1 \\
\hline 13 & 900170 & 1 & 900142 & 1 \\
\hline 14 & 900140 & 1 & 900191 & 2 \\
\hline 15 & 900142 & 1 & 900156 & 1 \\
\hline 16 & 900066 & 2 & 900090 & 2 \\
\hline 17 & 900651 & 2 & 900175 & 1 \\
\hline 18 & 900156 & 1 & 900117 & 2 \\
\hline 19 & 1000474 & 1 & 900082 & 2 \\
\hline 20 & 900393 & 2 & 900096 & 1 \\
\hline
\end{tabular}


1218 Rev. bras. zootec.

Tabela 6 - Classificação dos animais para a característica P550 (Geral), considerando os modelos uni e tricaracter

Table 6 - Animals classifications for trait P550 (General), considering the one and three-trait models

\begin{tabular}{|c|c|c|c|c|}
\hline \multirow[b]{2}{*}{$\begin{array}{l}\text { Classificação } \\
\text { Ranking }\end{array}$} & \multicolumn{2}{|c|}{$\begin{array}{c}\text { Unicaracter } \\
\text { One-trait }\end{array}$} & \multicolumn{2}{|c|}{$\begin{array}{l}\text { Tricaracter } \\
\text { Three-trait }\end{array}$} \\
\hline & Animal & $\begin{array}{l}\text { Sexo } \\
\text { Sex }\end{array}$ & Animal & $\begin{array}{l}\text { Sexo } \\
\text { Sex }\end{array}$ \\
\hline 1 & 800072 & 1 & 800072 & 1 \\
\hline 1 & 1000185 & 1 & 1000185 & 1 \\
\hline 2 & 1000191 & 1 & 1000191 & 1 \\
\hline 3 & 1000761 & 1 & 1000761 & 1 \\
\hline 4 & 1000011 & 1 & 1000186 & 2 \\
\hline 5 & 1000186 & 2 & 1000182 & 1 \\
\hline 6 & 1000182 & 1 & 900165 & 2 \\
\hline 7 & 1000474 & 1 & 900101 & 2 \\
\hline 8 & 1000183 & 1 & 900393 & 2 \\
\hline 9 & 1000190 & 1 & 1000939 & 1 \\
\hline 10 & 900165 & 2 & 1000273 & 2 \\
\hline 11 & 900177 & 1 & 1000183 & 1 \\
\hline 12 & 1000315 & 2 & 900177 & 1 \\
\hline 13 & 900101 & 2 & 1000190 & 1 \\
\hline 14 & 1000939 & 1 & 1000474 & 1 \\
\hline 15 & 900090 & 2 & 1000249 & 1 \\
\hline 16 & 900142 & 1 & 1000266 & 1 \\
\hline 17 & 1000216 & 1 & 1000315 & 2 \\
\hline 18 & 1000010 & 1 & 1000011 & 1 \\
\hline 19 & 1000771 & 1 & 900090 & 2 \\
\hline 20 & 900195 & 2 & 900142 & 1 \\
\hline
\end{tabular}

Em trabalho com gado de leite, TORRES (1998) encontrou valores de porcentagem de indivíduos comuns variando de 22,7 a $77,3 \%$, ao classificar os animais considerando ou não a existência de heterogeneidade de variância entre rebanhos, para características de produção de leite.

\section{Conclusões}

Os modelos mais adequados para estimação dos componentes de (co)variância, para peso aos 365 e 550 dias, de animais dos grupos genéticos formadores da raça Canchim, são aqueles que consideram a existência de variâncias específicas para cada grupos genéticos.

A utilização de modelos que não consideram a existência de variâncias específicas para cada grupo genético participante do processo de formação da raça Canchim não apresentou resultados que evidenciaram influência da existência destas variâncias na avaliação genética, principalmente considerando a característica peso aos 365 dias.

\section{Agradecimento}

À Empresa Brasileira de Pesquisa Agropecuária - Centro Nacional de Pesquisa Pecuária Sudeste, pelo fornecimento do conjunto de dados utilizado nas análises deste projeto.

\section{Referências Bibliográficas}

BOLDMAN, K.G., KRIESE, L.A., Van VLECK, L.D. et al. 1993. A manual for use of MTDFREML: a set of programs to obtain estimates of variances and covariances (DRAFT). Lincoln: Department of Agriculture/Agricultural Research Service. 120p.

CREWS, D.H., FRANKE, D.E. 1998. Heterogeneity of variances for carcass traits by percentage Brahman inheritance. J. Anim. Sci., 76:1803-1809.

De VEER, J. C., Van VLECK, L.D. 1987.Genetics parameters for first lactation milk yields at three levels of herd production. J. Dairy Sci., 70:(7):1434-1441.

DONG, M.C., MAO, I.L. 1990. Heterogeneity of (co)variance and heritability in different levels of intra-herd milk production variance and of herd average. J. Dairy Sci., 73(3):843-851.

ELER, J.P., LÔBO, R.B., ROSA, A.N. 1989. Influência de fatores genéticos e de meio em pesos de bovinos da raça Nelore criados no estado de São Paulo. R. Soc. Bras. Zootec., 18(2):112-123.

ELER, J.P., Van VLECK, L.D., FERRAZ, J.B.S. et al. 1995. Estimation of variances due to direct and maternal effects for growth traits of Nelore cattle. J. Anim. Sci., 73:3253-3258.

FREITAS, A.R., VENCOVSKY, R. 1993. Métodos de estimação de variâncias e parâmetros afins de características de crescimento em bovinos. Pesq. Agropec. Bras., 28(7):855-861.

GREGORY, K.E., CUNDIFF, L.V., KOCH, R.M. 1995. Genetic and phenotypic (co)variances for production traits of intact male populations of purebred and composite beef cattle. J. Anim. Sci., 73:2227-2234.

HENDERSON, C.R. 1975. Best linear unbiases estimation and prediction under a selection model. Biometrics., 31: 423-447.

IBAÑEZ, M.A., CARABAÑO, M.J., FOULLEY, J.L. et al. 1996. Heterogeneity of herd-period phenotypic variances in the Spanish Holstein-Friesian cattle: Sources of heterogeneity and genetic evaluation. Livest. Prod. Sci., 45:137-147.

LÔBO R.B., REYES, A., BEZERRA, L.A.F. et al. Parâmetros fenotípicos e genéticos de pesos e perímetro escrotal às idades-padrão em animais da raça Nelore. In: REUNIÃO ANUAL DA SOCIEDADE BRASILEIRA DE ZOOTECNIA, 32, 1995, Brasília. Anais ... Brasília: SBZ, 1995, p.625-627.

MAGNABOSCO, C.U., FAMULA, T.R., LÔBO, R.B. et al. Componentes de variância e covariância para características de crescimento em um rebanho da raça Nelore mocho do estado de São Paulo. In: REUNIÃO ANUAL DA SOCIEDADE BRASILEIRA DE ZOOTECNIA, 32, 1995, Brasília. Anais ... Brasília: SBZ, 1995. p.677-679.

MAGNABOSCO, C.U. Estimativas de parâmetros genéticos em características de crescimento de animais da raça Nelore usando os métodos de Máxima Verossimilhança Restrita e Amostragem de Gibbs. Ribeirão Preto, SP: USP, 1997, 83p. Tese (Doutorado em Ciências) - Universidade de São Paulo, 1997. 
MASCIOLI, A.S., ALENCAR, M.M., BARBOSA, P.F. et al. 1996. Estimativas de parâmetros genéticos e proposição de critérios de seleção para pesos na raça Canchim. R. Soc. Bras. Zootec., 25(1):72-82.

MEYER, K., HAMMOND, M.J., MACKINNON, M.J. et al. 1991. Estimates of covariances between reproduction and growth in Australian beef cattle. J. Anim. Sci., 69:3533-3543.

MOOD, A. M., GRAYBILL, F. A., BOES, D. C. 1974. Tests of hypotheses. In:___. Introduction to the theory of statistics, Tokio: McGraw-Hill. p.401-470.

NUNEZ-DOMINGUEZ, R., Van VLECK, L.D., CUNDIFF, L.V. 1995. Prediction of genetic values of sires for growth traits of crossbred cattle using a multivariate animal model with heterogeneous variances. J. Anim. Sci., 73:2940-2950.

RODRIGUEZ-ALMEIDA, Van VLECK, L.D., CUNDIFF, L.V. et al. 1995. Heterogeneity of variance by sire breed, sex, and Dam breed in 200 and 365-day weights of beef cattle from a top cross experiment. J. Anim. Sci., 73:2579-2588.

SAMPAIO I.B.M. 1998. Testes não paramétricos In:__Estatística aplicada à experimentação animal. Belo Horizonte: Fundação de Ensino e Pesquisa em Medicina Veterinária. p.192-205.

SPIEGEL, S. 1977. Medidas de correlação e suas provas de significância. In:__Estatística não-paramétrica para as ciências do comportamento. Tradução Alfredo Alves de Faria. São Paulo: MCGraw-Hill do Brasil Ltda. p.220-270.
SILVA, L.O.C., ROSA, A.N., NOBRE, P.R.C. et al. 1987. Análise de pesos de bovinos nelore criados a pasto no Estado de São Paulo, Brasil. Pesq. Agropec. Bras., 22(11):1245-1256.

TORRES, R.A. Efeito da heterogeneidade de variância na avaliação genética de bovinos da raça holandesa no Brasil. Belo Horizonte, MG:UFMG, 1998. 124p. Tese (Doutorado em Ciência Animal) - Universidade Federal de Minas Gerais, 1998.

VIANA, A.T., SANTIAGO, M., GOMES, F.P. 1962. Formação do gado Canchim pelo cruzamento Charolais-Zebu. Rio de Janeiro: Estudos Técnicos, ${ }^{\circ}{ }^{\circ}$ 19. SAI.

VINSON, W.E. 1987. Potential bias in genetic evaluations from differences in variation withim herds. J. Dairy Sci., 70(11):2450-2455.

WINKELMAN, A., SCHAEFFER, L.R. 1988. Effect of heterogeneity of variance on dairy sire evaluation. J. Dairy Sci., 71(11):3033-3039.

Recebido em: 29/12/99

Aceito em: 07/11/00 